\title{
Rietveld Analysis of Computed Tomography and its Application to Methanol to Olefin Reactor Beds
}

Authors

\section{David Stephen Wragga*, Matthew G. O'Brien ${ }^{\mathrm{bb}}$, Marco Di Michiel ${ }^{\mathrm{c}}$ and Francesca Lønstad- Bleken $^{\text {d }}$}

${ }^{\mathrm{a}}$ InGAP Centre for reserach Based Innovation, Department of Chemistry, University of Oslo, PO Box 1033 Blindern, Oslo, 0315, Norway

bInorganic Chemistry, University of Utrecht, Universiteitsweg 99, Utrecht, 3584, The Netherlands

c The European Synchrotron, CS 40220, Grenoble Cedex 9, 38043, France

${ }^{\mathrm{d}}$ SINTEF Materials and Chemistry, SINTEF, Forskningsveien 1, Oslo, 0314, Norway

Correspondence email: david.wragg@smn.uio.no

Synopsis Tomographic reconstructions of zeolite catalyst beds using structural data extracted by parametric Rietveld refinement show small but significant 3D variations in the degree of catalyst deactivation after reaction.

Abstract We have created tomographic reconstructions giving three dimensional data on the distribution of various structural features for SAPO-34 zeolite catalyst beds used in the commercially important methanol to olefin (MTO) conversion process. The data were processed using parametric Rietveld refinement to treat entire slices of the tomograph as single refined datasets, allowing extraction of real structural parameters from all voxels of the reconstruction. This has the advantage over more traditional methods of XRD-computed tomography using peak intensities, that the structural parameters are independent of the intensity, meaning that information can still be extracted from poor datasets: an example is shown where part of the sample was no longer in the beam during data collection. Reconstructions using several structural parameters are presented and the results compared. Analysis of the variation of the catalyst $c$-axis (linked to degree of deactivation in earlier work) shows small but significant 3D variations in the degree of deactivation with patterns which depend on the silicon content of the catalyst. Average data for the tomographic slices compare well with the results of earlier in operando 2D reactor scanning experiments.

\section{Introduction}

In the quest to understand catalytic materials, the variety and location of their active sites under working conditions, it is essential to have in situ data with both time and space resolution (Buurmans \& Weckhuysen, 2012, Beale et al., 2010), i.e. to see where the catalyst is active and when. X-ray 
computed tomography, the development of which was first driven by medical imaging applications, is now recognized as an important technique in the study of all kinds of materials (Hounsfield, 1973, Lauterbur, 1973, Harding et al., 1987, Arnberg \& Mathiesen, 2007, Bertrand et al., 2012, Adams, 2013). X-ray diffraction computed tomography (XRD-CT) (Harding \& Kosanetzky, 1989) is becoming a broadly applicable technique for studying the 3-dimensional structure of catalysts, both as individual bodies and beds (Beale et al., 2010). It is now possible to collect data fast enough to carry out time resolved tomographic studies of reactions with XRD (Jacques et al., 2011). However, so far most of the analysis carried out has been based on simple observations from the diffraction patterns, for example, phase identification and quantification or crystallite size analysis from single peaks; or analysis of the total integrated intensity over a selected Q-range (Harding et al., 1990, Hall et al., 1996, Hall et al., 1998, Espinosa-Alonso et al., 2009, Lazzari et al., 2009, Jacques et al., 2011, Alvarez-Murga et al., 2011, O'Brien et al., 2012, Álvarez-Murga et al., 2012, Alvarez-Murga et al., 2012, Voltolini et al., 2013). Bleuet et al reported Rietveld analysis of selected data points in an XRD tomographic reconstruction in 2009 (Bleuet et al., 2008). Palancher et al have shown that it is possible to carry out Rietveld quantitative analysis on a complete, sub-micrometer resolution, tomographic dataset (Palancher et al., 2011) and Scarlett et al have carried out parametric Rietveld quantitative analysis on energy dispersive XRD tomographic data but did not reconstruct 3-dimensional images of their electrochemical cells (Scarlett et al., 2009), although they were able to measure electrode thickness as a function of time from the data. De Nolf and co-workers have recently developed dedicated software (XRDUA) for full profile quantitative analysis and tomographic reconstruction using powder diffraction data, especially from non-ideal powders (i.e. powders where the Debye rings contain poorly averaged Bragg spots due to large single crystals in the sample) (De Nolf et al., 2014). Egan et al have $r$ used Le Bail fitting methods to perform tomographic reconstructions of teeth based on the lattice parameters, strain and preferred orientation of the phases present (Egan et al., 2013). Korsunsky et al used full profile methods to extract lattice parameters and thereby study strain in XRD-CT reconstructions of aeronautical nickel alloy and dental prostheses (Korsunsky et al., 2006, Korsunsky et al., 2011). Recent work by Bonnin et al has also combined and compared Rietveld analysis of phases in a tomographic reconstruction with grain indexing to give the orientations of the individual crystallites in the sample from "spotty” 2D XRD patterns (Bonnin et al., 2014). Jacques et al have shown the validity of pair distribution function analysis for tomographic data; obtaining crystallite size and phase distribution data from working catalysts (Jacques et al., 2013), a very interesting contribution which opens up the possibility of studying none crystalline materials and nanosized particles.

Here we present crystal structure analysis of complete XRD-CT reconstructions of catalyst beds used in the industrially important methanol to olefin (MTO) conversion process (Barger, 2002) using parametric Rietveld methods. These methods have allowed us to refine atom positions, link related 
parameters across all the powder patterns in the tomographic datasets and make the whole process faster and easier.

The catalyst used in the MTO process is the zeolite-type framework SAPO-34. SAPO-34 is derived from the AlPO-34 structure: a rhombohedral (spacegroup R-3, $a \approx 13.85, c \approx 14.8 \AA$ in the hexagonal setting used here; chabazite zeolite framework) open framework made up of aluminium and phosphorus atoms tetrahedrally coordinated by oxygen. The framework forms large internal cages (chabazite cages in the zeolite nomenclature) which can be accessed through windows of 6 and 8 tetrahedral atoms (figure 1).

Figure 1.

To make AlPO-34 catalytically active in the MTO process we replace some of the phosphorus atoms with silicon. In order to balance the charge of the framework when Si4+ replaces $\mathrm{P} 5+$, protons are coordinated to the framework. These "acid sites" help to convert methanol into hydrocarbons inside the cages, the \% of silicon substituted into the tetrahedral sites is therefore commonly used as an indicator of acidity (up to $8 \%$ silicon substitution can give a structure with no Si-O-Si linkages and therefore 1 acid site per silicon in the framework) (Stöcker, 1999). The size of the windows controls the products which can leave the cages (Hereijgers et al., 2009) making the catalyst very selective for the olefins ethene, propene and butene- high value chemical building blocks. The structure which makes SAPO-34 such an effective catalyst for the MTO process also leads to its most significant problem: the cages, after a time, fill with aromatic hydrocarbons which are too large to escape through the 8-tetrahedral atom windows (coke) and this deactivates the catalyst. The catalyst can be regenerated by burning out the hydrocarbon coke at high temperature (calcination), but this requires MTO plants to utilise expensive and complex circulating bed technology and may be connected to the long term deactivation of the catalyst due to loss of acidity (Barger, 2002). The link between filling of the cages, deactivation of the catalyst and expansion of the crystal structure was first observed in in operando experiments using a capillary microreactor (Wragg et al., 2009) and has since been studied for other frameworks (Wragg et al., 2011, Wragg, Bleken, et al., 2013). The overall aim of our studies of this material is to extend the active lifetime of the industrial catalyst and reduce the long term loss of acidity.

The structural parameters obtained from the refinements carried out here on the XRD-CT data appear to show preferred reactant paths through the bed, leading to varying degrees of deactivation in different parts of the bed. The tomographic approach reveals variations in the catalyst structure within depth slices of the bed as well as average trends from inlet to outlet which can be compared with the previously reported in situ z-scan data on this reaction (Wragg et al., 2012). Such variations may be significant for future test reactor designs. Furthermore, Rietveld-CT allows us to extract information 
from datasets which would be extremely hard to analyse with conventional XRD-CT methods, by imaging with parameters which are not correlated with the overall intensity.

\section{Experimental}

SAPO-34 Catalysts were obtained from SINTEF Oslo (prepared by the method of Wendelbo et al (Wendelbo et al., 1998)) and pressed and sieved to a particle size of between 0.25 and $0.42 \mathrm{~mm}$. Tomographic data were collected at the high energy beamline ID15B of the ESRF. The catalyst beds (100 mg catalyst) were prepared in a $4 \mathrm{~mm}$ internal diameter glass tubes and exposed to a flow of helium gas bubbled through methanol at $19.5^{\circ} \mathrm{C}$ (flowing from bottom to top). The flow rate was 50 $\mathrm{ml} \mathrm{min}{ }^{-1}$. The reactor was heated with a pair of Leister LE Mini heat guns and the reaction temperature varied between $346^{\circ} \mathrm{C}$ at the top of the catalyst bed to $338^{\circ} \mathrm{C}$ at the bottom. The reactor tube and heat guns were mounted on a Huber stage capable of rotation and translation in the $\mathrm{x}, \mathrm{y}$ and $\mathrm{z}$ directions and a Pixium area detector was used. The sample to detector distance was calibrated at $1473 \mathrm{~mm}$ using NIST SRM660a lanthanum hexaboride and the wavelength was $0.14257 \AA$ (89.965 $\mathrm{keV}$ ). Prior to collection of the XRD-CT data the reaction gas flow was switched to pure helium and the reactor was cooled to room temperature as rapidly as the heat guns would allow. The exposure time was 1 second per frame and collection of a dataset suitable for tomographic reconstruction took around 7 hours. The series of 2D diffraction images were radially integrated to $1 \mathrm{D}$ powder patterns using the program DATASQUEEZE, these raw diffraction images were used for tomographic reconstruction in the program Snark93 (Browne et al., 1993). The data collection strategy and methods of reconstruction are described in detail elsewhere (Jacques et al., 2011, O'Brien et al., 2012). 5 slices of tomographic data, evenly spaced from the reactor inlet to the top of the bed, were collected for each sample, with a beam size of $0.1 \mathrm{~mm}$. The approximate locations of the five slices are shown in supporting figure S1.

The reconstructed datasets now consisted of a set of powder XRD patterns, each representing the total diffraction from a $0.1 \mathrm{~mm}$ cubic voxel in the tomographic reconstruction. These patterns were refined using the parametric Rietveld method (Stinton \& Evans, 2007) with the program TOPAS 4.2 (Coelho, 2006). The stability of the refinement method is such that it was possible to refine each slice of the reconstruction as a complete dataset, including the empty volumes and the glass sides of the reactor. Further information on the fitting process is given in the supporting information. We refer to this reconstruction hereafter as the Rietveld-CT of the data.

The structural model used is described in earlier publications (Wragg et al., 2011, Wragg, Groenvold, et al., 2013, Wragg et al., 2009, Wragg et al., 2010, Wragg et al., 2012, Zokaie et al., 2013) and uses "dummy" carbon atom positions to estimate the occupancy of the SAPO-34 cages (i.e. the hydrocarbon coke which builds up in the cages and deactivates the catalyst during the MTO process) and help prevent the framework structure collapsing during refinement due to the electron density in 
the cages. The refinements were split into tomographic slices of $50 \times 50$ voxels, i.e. 2500 powder patterns were refined simultaneously as a single surface of Rietveld data. For the fully reacted $8 \% \mathrm{Si}$ sample a 53 x 53 voxel area was refined as the sample moved slightly during data collection and some sections were outside the initial 50 x 50 range. The zero error, tan- $\theta$ sample broadening term (O'Brien et al., 2011), scale factor and quartz background peak position (see background treatment below) were refined as single parameters applied to all patterns in each slice (thus applying a constant, but refined line position shift to all patterns in each tomographic slice as discussed by Palancher et al (Palancher et al., 2011)). No preferred orientation corrections were applied as earlier flat plate PXRD studies of SAPO-34 by various authors (see Stöcker’s review of MTO chemistry) (Stöcker, 1999) show no sign preferred orientation effects when compared to capillary measurements.

Two isotropic thermal parameters (Biso) were refined in each powder pattern one for the tetrahedral atoms (Al/Si/P; T-atoms) and one for oxygen. Maximum and minimum limits of 10 and 1 were placed on these parameters. Refinement with Biso fixed across all powder patterns for the two atom groups was also carried out, based on the assumption that thermal factors should be equal as the atoms are at the same temperature everywhere in the cooled reactor bed. Such an approach is not without precedent; Agostini et al used parametric methods to improve the refinement of site occupancies during zeolite template removal by parameterising a linear variation of atomic positions with temperature (Agostini et al., 2009). The coordinates of the T-atoms were also refined. Refinement of the oxygen positions was not possible, leading to collapse of the framework structure when attempted. This is probably due to the incomplete structural model with only three "dummy" carbon atoms accounting for all of the electron density inside the cages after reaction. Restraints on the framework bond lengths and angles were used to prevent the T-atoms migrating into the cages to fit diffuse areas of electron density not accounted for by the model. The background was fitted with a 7-term Chebyshev polynomial and a broad peak used to fit the background "bump" from the quartz tube at $\sim 18^{\circ} 2 \theta$. This allows us to study the lattice parameters, strain broadening, overall scale, $\mathrm{T}$ atom positions, cage occupancies and numerous other modes of tomographic projection of the data from the SAPO-34 structure. In this case we concentrate mainly on the $c$-axis variation which has been clearly linked with development of reaction intermediates and coke (Wragg et al., 2009, Wragg et al., 2012, Zokaie et al., 2013, Wragg, Groenvold, et al., 2013), however, information from some of the other parameters is considered. A full list if refined parameters for the three samples, detailing whether they were refined against individual powder patterns in the dataset or all data, is given in table S1 of the supporting information.

\section{Results}

We have studied three tomographic datasets representing different stages of the MTO process and different silicon contents in the catalyst. The silicon content is significant as this determines the 
acidity of the catalyst (the number of active sites), a crucial parameter in its effectiveness in the MTO process (Bleken et al., 2009). The first two samples contained 8 \% silicon (determined by energy dispersive X-ray spectroscopy in an FEI scanning electron microscope). The first of these was subjected to methanol flow for 5 minutes before rapid cooling to room temperature, while the second was used for MTO conversion for 195 minutes before cooling and collection of XRD-CT data. The third sample contained $4 \% \mathrm{Si}$ and was used in the MTO reaction for 230 minutes prior to cooling and data collection. Our diffraction data are therefore comparable not with the in situ data reported in our earlier work (Wragg et al., 2009, Wragg et al., 2010, Wragg et al., 2011, Wragg et al., 2012, Wragg, Groenvold, et al., 2013) but with the ex situ XRD studied by Zokaie et al and Wragg et al (Zokaie et al., 2013, Wragg, Groenvold, et al., 2013). We note however, that the data presented in the latter two references indicate very similar trends in the variation of the crystal structure with increasing duration of MTO reaction time to the in situ data.

\subsection{SAPO-34, $8 \% \mathrm{Si}$, Quenched}

The five 2-dimensional slices of the Rietveld-CT reconstruction using $c$-axis length for the $8 \% \mathrm{Si}$ sample quenched after 5 minutes of MTO reaction are shown in figure 2 as colour contour plots. The circular profile of the reactor tube is clearly visible.

Fig. 2

The shift in $c$-axis length from the initial value of $14.6 \AA$ for the freshly calcined sample is relatively small (this axis can extend to over $15.1 \AA$ in similar samples (Wragg et al., 2009, Wragg et al., 2012)), with a maximum $c$ value of $14.8 \AA$; as expected for a catalyst subjected to a short time on stream. However, we see significant variations within the range both with increasing distance from the inlet point and across the individual slices. The extension of the $c$-axis averaged across each slice drops slightly from slice (a) (the inlet end of the bed) to slice (b) then increases to the outlet at slice (e). Examining the slices reveals a pattern of greater expansion at the right edge of the reactor tube. The $c$-axis variation of specific voxels seems to follow through the series of slices (i.e. if voxel (35, $35)$ in slice (c) has a large c-axis compared to surrounding voxels, so will the $(35,35)$ voxel in slice (e)) suggesting that the cause of expansion (i.e. the reagents which cause coking of the catalyst) is spreading in a predictable way up the bed. Figure 1(f) shows the c-axis patterns vs slice number in several voxels compared to the averaged value for each slice. The greater c-axis extension at the edge of the reactor may be due to the fact that this area was closest to the heat guns used to maintain the temperature, however, the fact that this expansion is only observed on one side of the reactor, while heat guns were positioned on opposite sides of the reactor perpendicular to the X-ray beam, does not support this proposition.

To check that the patterns observed in the Rietveld-CT are not simply due to variations in the quality of the refinement we compared the data for the $c$-axis reconstruction to reconstructions based on Rwp 
(indicating the quality of Rietveld fit). The Rwp reconstruction of the quenched $8 \%$ Si sample is shown in figure 3 and clearly does not show the patterns seen in the $c$-axis tomograph, indeed, the distribution of Rwp in the discs of the reactor slices appears to be essentially random. The Rwp values may appear rather high (average Rwp decreases from $21.3 \%$ to $20.1 \%$ from slice (a) to slice (e), see supporting information, figure S2), however, the Rexp (expected minimum R-value) values for the reconstructed data are rather high (in the region of $18 \%$ for most of the voxels)- this is due to the incorrect assumption in TOPAS (common to most Rietveld software) that the errors in the intensities for PXRD data collected with an area detector are equal to the square root of the intensity (real errors are related to the number of detector pixels under the powder rings, which depend on detector region), and may also be due to the unusual counting statistics in the reconstructed data (Rexp for the raw data patterns is around 10-15 \%). A typical Rietveld fit from the dataset (voxel 35, 35 in layer 0) is shown in figure 3(f).

Fig. 3

If we rule out variations in the fit quality and temperature inconsistencies, our best current explanation for the 3-D variations seen in the $c$-axis reconstruction of this sample is that they represent preferred pathways through the reactor bed. We have demonstrated elsewhere (Wragg et al., 2012) that the longer the reactants take to pass through the fixed bed MTO reactor the more likely they are to form large hydrocarbon molecules in the cages, leading to increased $c$-axis extension, it may therefore be assumed that the reaction gases passed more quickly through the areas of the bed with a shorter $c$ axis. This may be due to several factors including the packing of the catalyst particles in the bed, which can lead to "channelling” of the reagents through certain parts of the bed in a packed bed reactor (Fogler, 1999). The side walls of the reactor can also have an influence on flow rate in some circumstances (Chorkendorff \& Niemantsverdriet, 2007).

Further reconstructions based on Biso and coordinate values for aluminium and phosphorus; and peak broadening were also carried out for this dataset, however, the results did not show any interesting patterns, see supporting information (figure S3, S4, S5). It is possible that the Biso values obtained are not entirely reliable due to the slightly limited Q-range available in the data and the poor quality of the data at higher Q-values. The lack of variation in the peak broadening plots suggests that, despite the earlier observation that this parameter seems to be linked to coking of the catalyst (Wragg et al., 2009), it is not affected significantly enough by the process for the variations to be visible in the tomograms.

\subsection{SAPO-34, $8 \% \mathrm{Si}$, Fully Reacted}

A very similar tomograph is observed in the fully reacted $8 \%$ silicon sample (figure 4 a-e). Here the $c$-axis values observed are longer (between 14.7 and $15.05 \AA$ ) as we would expect from the literature (Wragg et al., 2009, Zokaie et al., 2013). As for the previous sample we can see patterns being 
passed through equivalent voxels in the slices, especially in the top 3 slices in areas where $c$-axis expansion is well established, e.g. voxel 015_041 (figure 4 f). Again the expansion at the edges the reactor in the slices furthest from the inlet may be linked to the close proximity of the heat gun, but is only observed on one side of the reactor.

Fig. 4

During data collection on this sample the catalyst bed moved in the reactor leaving an empty space on the left hand side of slices 3 and 4 . This caused problems in scaling which made the data from ordinary tomographic reconstructions using intensity in a set Q-range almost useless (figure 5). Using Rietveld-CT reconstruction however, we obtain almost as much information as for the other samples as the lattice parameters can be extracted regardless of the intensity. Such information would be difficult to extract using the batch Le Bail type fits which have been applied to other tomographic data due to the massive scale differences.

Fig. 5

It is also interesting to look at the $a$-axis reconstruction of this sample, to check that data really are recovered correctly despite the void area in the reconstruction. The patterns observed in the slices are similar to those seen in the $c$-axis reconstruction (figure 6 (a-e)) but the magnitude of the $a$-axis variation is relatively small and the average values in adjacent slices are within one standard deviation of each other (figure 6(f)). It is therefore not possible to point to a trend in the average $a$-axis values from these data.

Fig. 6

Tomographic slices reconstructed on cage occupancy for fully reacted $8 \%$ Si SAPO-34 are shown in supporting figure S6. These slices show a significant similarity to the $a$-axis reconstructions, reinforcing the earlier observation that the crystal structure changes (in particular the lattice parameter variations) during the MTO process are related to the filling of the chabazite cages with organic coke during the reaction. Figure 7 shows the patterns in reconstructions on the $a$ and $c$-axes and cage occupancy for slice 0 of the fully reacted $8 \%$ silicon bed side by side for easy comparison.

Fig 7

\subsection{SAPO-34, $4 \% \mathrm{Si}$, Fully Reacted}

A second fully reacted sample containing $4 \%$ silicon shows a different pattern across the slices (caxis reconstruction, along with selected voxel and average $c$-axis plots through the slices, is shown in figure 8). In this case we do not see large areas of high $c$-axis expansion at the edge of the reactor; however, patterns can still be seen in equivalent voxels through the slices, with small areas of $c$-axis extension spread around the slices. There is no sign of greater $c$-axis extension close to the reactor 
edges in this sample, giving further support to the theory that the expansion observed at the edge of the reactor in the $8 \%$ Si sample is not due to overheating.

The pattern of the average $c$-axis value through the 5 slices is very similar to that observed for the quenched $8 \%$ Si sample with a small range of values and small standard deviations in the average, however the overall values of $c$ for this sample are the largest of any of the datasets (see figure 9 below). As for the other two samples the second slice of the tomograph has the smallest average $c$ axis value.

Fig. 8, 9

Plotting the average $c$ value from each slice (with standard deviation bars to indicate the range of values present in each slice) on the same axes shows a similar pattern for the three samples (figure 8), but with a much larger variation in the fully reacted $8 \%$ silicon sample, both in terms of the changes in $c$ between slices and the variation within each slice (as expressed by the error bars in the graphs). The quenched sample shows similar values for the first two slices with a gradual increase from slice (b) to slice (e). In the fully reacted $8 \%$ Si sample the c-axis length decreases significantly (i.e. beyond the standard deviation in the average) from slice (a) to slice (b) before increasing strongly towards slice (e). This recalls the pattern seen for this sample in fast z-scan experiments (Wragg et al., 2012), in which a relatively flat profile of $c$-axis values along the bed early in the experiment develops into a profile with an obvious step at a height of around $3 \mathrm{~mm}$ from the reactor inlet after approximately 90 minutes. Graphs showing a direct comparison of the Rietveld-CT and z-scan data are shown in the supporting information (Figure S7).

The smallest variation in average $c$-axis lengths through the bed slices is seen for the fully reacted $4 \%$ silicon sample. Again, this is in agreement with the in situ z-scan data published previously. The reduced variation is attributed to the smaller number of active catalytic sites in the lower silicon material, which leads to a slower onset of deactivation. In fact this sample seems to be more like the quenched $8 \%$ silicon sample than the fully reacted $8 \%$ silicon SAPO-34. Looking at the average cage occupancies (figure 10) for each sample we see that the average levels of electron density in the cages for the $4 \%$ Si sample are also much closer to those observed for quenched $8 \%$ Si SAPO-34 than fully reacted $8 \%$ Si SAPO-34. The apparent decrease in average cage occupancy from the inlet to the outlet for the $4 \% \mathrm{Si}$ and quenched $8 \% \mathrm{Si}$ samples is unexpected, but may be due to outlying values having a large effect on the average (note the large standard deviations in the average occupancy values for the $4 \%$ Si sample, which also shows the strongest decline in cage occupancy).

Fig. 10

\section{Conclusions}


We have demonstrated the feasibility of analysing XRD-CT data using the Rietveld method by reconstructing three SAPO-34 catalyst beds using several different, real structural parameters. This allows us to track the development of coke in different parts of the reactor, observing small 3dimensional variations in catalyst deactivation. Furthermore we have demonstrated that Rietveld-CT can extract meaningful information from XRD-CT datasets which would be very difficult to study with conventional reconstruction techniques.

In general we see that the coke from the MTO reaction is more evenly spread around the sample containing $4 \%$ silicon, this suggests that catalysts with this level of silicon will probably be the most long-lived and effective MTO catalysts. The existence of areas in the fixed bed reactors with significantly different levels of deactivation in the $8 \%$ silicon catalysts examined indicates that care should be taken when we study the behaviour of SAPO-34 in reactors of this type, in order to avoid flow problems. The variations in the average $c$-axis values with reactor position recall the reduction observed in the $c$-axis length close to the reactor inlet at later stages of the MTO reaction in the in situ Z-scanning experiments (Wragg et al., 2012). We also note that there seems to be some correlation between the $a$-axis and cage occupancy reconstructions, which may explain the origins of the structural variations. Since less 3D variation in the lattice parameters is observed for the $4 \%$ silicon sample we believe that temperature inconsistencies in the beds do not make a significant contribution to the variations seen in the $c$-axis of SAPO-34 with $8 \%$ silicon and the variations observed are probably due to preferred pathways through the catalyst bed.

Rietveld CT could be applied very easily to other well-ordered catalyst systems which exhibit good crystallinity and the combination of the technique with the very fast tomographic data collection now possible (O'Brien et al., 2009, O'Brien et al., 2012, Jacques et al., 2013) makes in situ Rietveld-CT a real possibility.

Acknowledgements We acknowledge the ESRF for providing beam time at ID15B and the inGAP centre for research-based innovation for funding.

\section{References}

Adams, J. E. (2013). Nat Rev Endocrinol 9, 28-42.

Agostini, G., Lamberti, C., Palin, L., Milanesio, M., Danilina, N., Xu, B., Janousch, M. \& van Bokhoven, J. A. (2009). J. Am. Chem. Soc. 132, 667-678.

Álvarez-Murga, M., Bleuet, P., Garbarino, G., Salamat, A., Mezouar, M. \& Hodeau, J. L. (2012). Physical Review Letters 109, 025502.

Alvarez-Murga, M., Bleuet, P. \& Hodeau, J.-L. (2012). J. Appl. Cryst. 45, 1109-1124.

Alvarez-Murga, M., Bleuet, P., Marques, L., Lepoittevin, C., Boudet, N., Gabarino, G., Mezouar, M. \& Hodeau, J.-L. (2011). J. Appl. Cryst. 44, 163-171.

Arnberg, L. \& Mathiesen, R. (2007). JOM 59, 20-26.

Barger, P. (2002). Zeolite for Cleaner Technologies, edited by M. Guisnet \& J.-P. Gilson, pp. 239-260. London: Imperial College Press.

Beale, A. M., Jacques, S. D. M. \& Weckhuysen, B. M. (2010). Chem. Soc. Rev. 39, 4656-4672. 
Bertrand, L., Cotte, M., Stampanoni, M., Thoury, M., Marone, F. \& Schöder, S. (2012). Physics Reports 519, 51-96.

Bleken, F., Bjørgen, M., Palumbo, L., Bordiga, S., Svelle, S., Lillerud, K.-P. \& Olsbye, U. (2009). Top. Catal. 52, 218-228.

Bleuet, P., Welcomme, E., Dooryhee, E., Susini, J., Hodeau, J.-L. \& Walter, P. (2008). Nat Mater 7, 468-472.

Bonnin, A., Wright, J. P., Tucoulou, R. \& Palancher, H. (2014). Applied Physics Letters 105, 084103.

Browne, J. A., Herman, G. T. \& Odhner, D. (1993). SNARK93: A Programming System for Image Reconstruction from Projections. University of Pennsylvania.

Buurmans, I. L. C. \& Weckhuysen, B. M. (2012). Nat Chem 4, 873-886.

Chorkendorff, I. \& Niemantsverdriet, J. W. (2007). Concepts of Modern Catalysis and Kinetics, 2nd ed. Weinheim: Wiley-VCH.

Coelho, A. A. (2006). TOPAS V4.1, Bruker AXS.

De Nolf, W., Vanmeert, F. \& Janssens, K. (2014). J. Appl. Cryst. 47, 1107-1117.

Egan, C. K., Jacques, S. D. M., Di Michiel, M., Cai, B., Zandbergen, M. W., Lee, P. D., Beale, A. M. \& Cernik, R. J. (2013). Acta Biomaterialia 9, 8337-8345.

Espinosa-Alonso, L., O'Brien, M. G., Jacques, S. D. M., Beale, A. M., de, J., Barnes, P. \& Weckhuysen, B. M. (2009). J. Am. Chem. Soc. 131, 16932-16938.

Fogler, H. S. (1999). Elements of Chemical Reactor Engineering, 3rd ed. New Jersey: Prentice Hall. Hall, C., Barnes, P., Cockcroft, J. K., Colston, S. L., Häusermann, D., Jacques, S. D. M., Jupe, A. C. \& Kunz, M. (1998). Nuclear Instruments and Methods in Physics Research Section B: Beam Interactions with Materials and Atoms 140, 253-257.

Hall, C., Barnes, P., Cockcroft, J. K., Jacques, S. D. M., Jupe, A. C., Turrillas, X., Hanfland, M. \& Hausermann, D. (1996). Analytical Communications 33, 245-248.

Harding, G. \& Kosanetzky, J. (1989). Nuclear Instruments and Methods in Physics Research Section A: Accelerators, Spectrometers, Detectors and Associated Equipment 280, 517-528.

Harding, G., Kosanetzky, J. \& Neitzel, U. (1987). Medical Physics 14, 515-525.

Harding, G., Newton, M. \& Kosanetzky, J. (1990). Physics in Medicine and Biology 35, 33.

Hereijgers, B. P. C., Bleken, F., Nilsen, M. H., Svelle, S., Lillerud, K.-P., Bjørgen, M., Weckhuysen, B. M. \& Olsbye, U. (2009). J. Catal. 264, 77-87.

Hounsfield, G. N. (1973). The British Journal of Radiology 46, 1016-1022.

Jacques, S. D. M., Di Michiel, M., Kimber, S. A. J., Yang, X., Cernik, R. J., Beale, A. M. \& Billinge, S. J. L. (2013). Nat Commun 4.

Jacques, S. D. M., Di, M. M., Beale, A. M., Sochi, T., O'Brien, M. G., Espinosa-Alonso, L., Weckhuysen, B. M. \& Barnes, P. (2011). Angew. Chem., Int. Ed. 50, 10148-10152, S10148/10141-S10148/10111.

Korsunsky, A. M., Baimpas, N., Song, X., Belnoue, J., Hofmann, F., Abbey, B., Xie, M., Andrieux, J., Buslaps, T. \& Neo, T. K. (2011). Acta Materialia 59, 2501-2513.

Korsunsky, A. M., Vorster, W. J. J., Zhang, S. Y., Dini, D., Latham, D., Golshan, M., Liu, J., Kyriakoglou, Y. \& Walsh, M. J. (2006). Acta Materialia 54, 2101-2108.

Lauterbur, P. C. (1973). Nature 242, 190-191.

Lazzari, O., Jacques, S., Sochi, T. \& Barnes, P. (2009). Analyst 134, 1802-1807.

O'Brien, M. G., Beale, A. M., Jacques, S. D. M., Di Michiel, M. \& Weckhuysen, B. M. (2011). Applied Catal. A. 391, 468-476.

O'Brien, M. G., Beale, A. M., Jacques, S. D. M., Di, M. M. \& Weckhuysen, B. M. (2009). ChemCatChem 1, 99-102.

O'Brien, M. G., Jacques, S. D. M., Michiel, M. D., Barnes, P., Weckhuysen, B. M. \& Beale, A. M. (2012). Chem. Sci. 3, 509-523.

Palancher, H., Tucoulou, R., Bleuet, P., Bonnin, A., Welcomme, E. \& Cloetens, P. (2011). J. Appl. Cryst. 44, 1111-1119.

Scarlett, N. V. Y., Madsen, I. C., Evans, J. S. O., Coelho, A. A., McGregor, K., Rowles, M., Lanyon, M. R. \& Urban, A. J. (2009). J. Appl. Cryst. 42, 502-512.

Stinton, G. W. \& Evans, J. S. O. (2007). J. Appl. Cryst. 40, 87-95.

Stöcker, M. (1999). Microporous Mesoporous Mater. 29, 3-48. 
Voltolini, M., Dalconi, M. C., Artioli, G., Parisatto, M., Valentini, L., Russo, V., Bonnin, A. \& Tucoulou, R. (2013). J. Appl. Cryst. 46, 142-152.

Wendelbo, R., Akporiaye, D. E., Andersen, A., Dahl, I. M., Mostad, H. B., Fuglerud, T. \& Kvisle, S. (1998).

Wragg, D. S., Akporiaye, D. \& Fjellvåg, H. (2011). J. Catal. 279, 397-402.

Wragg, D. S., Bleken, F. L., O'Brien, M. G., Di Michiel, M., Fjellvag, H. \& Olsbye, U. (2013). Physical Chemistry Chemical Physics 15, 8662-8671.

Wragg, D. S., Groenvold, A., Voronov, A., Norby, P. \& Fjellvaag, H. (2013). Microporous Mesoporous Mater. 173, 166-174.

Wragg, D. S., Johnsen, R. E., Balasundaram, M., Norby, P., Fjellvåg, H., Grønvold, A., Fuglerud, T., Hafizovic, J., Vistad, Ø. B. \& Akporiaye, D. (2009). J. Catal. 268, 290-296.

Wragg, D. S., Johnsen, R. E., Norby, P. \& Fjellvåg, H. (2010). Microporous Mesoporous Mater. 134, 210-215.

Wragg, D. S., O'Brien, M. G., Bleken, F. L., Di Michiel, M., Olsbye, U. \& Fjellvåg, H. (2012). Angew. Chem. Int. Ed. Engl. 51, 7956-7959.

Zokaie, M., Wragg, D. S., Grønvold, A., Fuglerud, T., Cavka, J. H., Lillerud, K. P. \& Swang, O. (2013). Microporous Mesoporous Mater. 165, 1-5. 
Figure 1 The crystal structure of SAPO-34 framework projected along the $a$-axis (left), showing the 8-tetrahedral atom windows. The cage, with 8 and 6 T-atom windows, is shown on the right. Oxygen atoms are omitted for clarity.
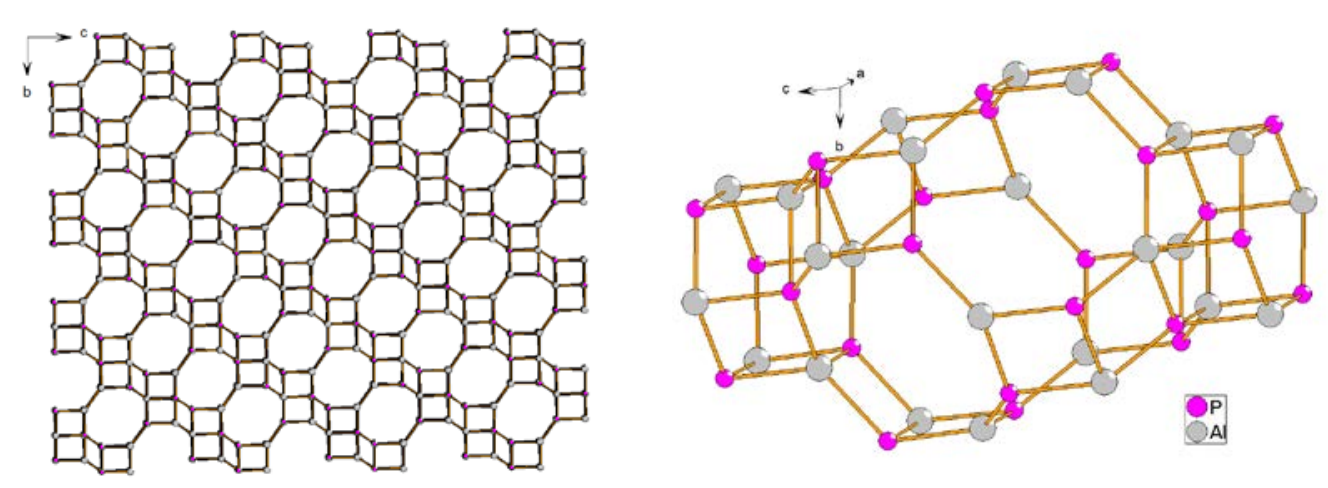

Figure 2 (a-e) Colour contour plots of the $c$-axis Rietveld-CT reconstruction of a SAPO-34 (8 \% Si) reactor bed used in the MTO process for 5 minutes. The tomograph consists of five slices 2-D from slice (a) (inlet) to slice (e) (outlet). The colour scale used to indicate the $c$-axis expansion is shown in the top right corner. (f) is a plot of c-axis values for selected voxels in each slice (dotted lines) with the average value (heavy line) for comparison. Slice numbers 0-4 in (f) correspond to slices a-e.
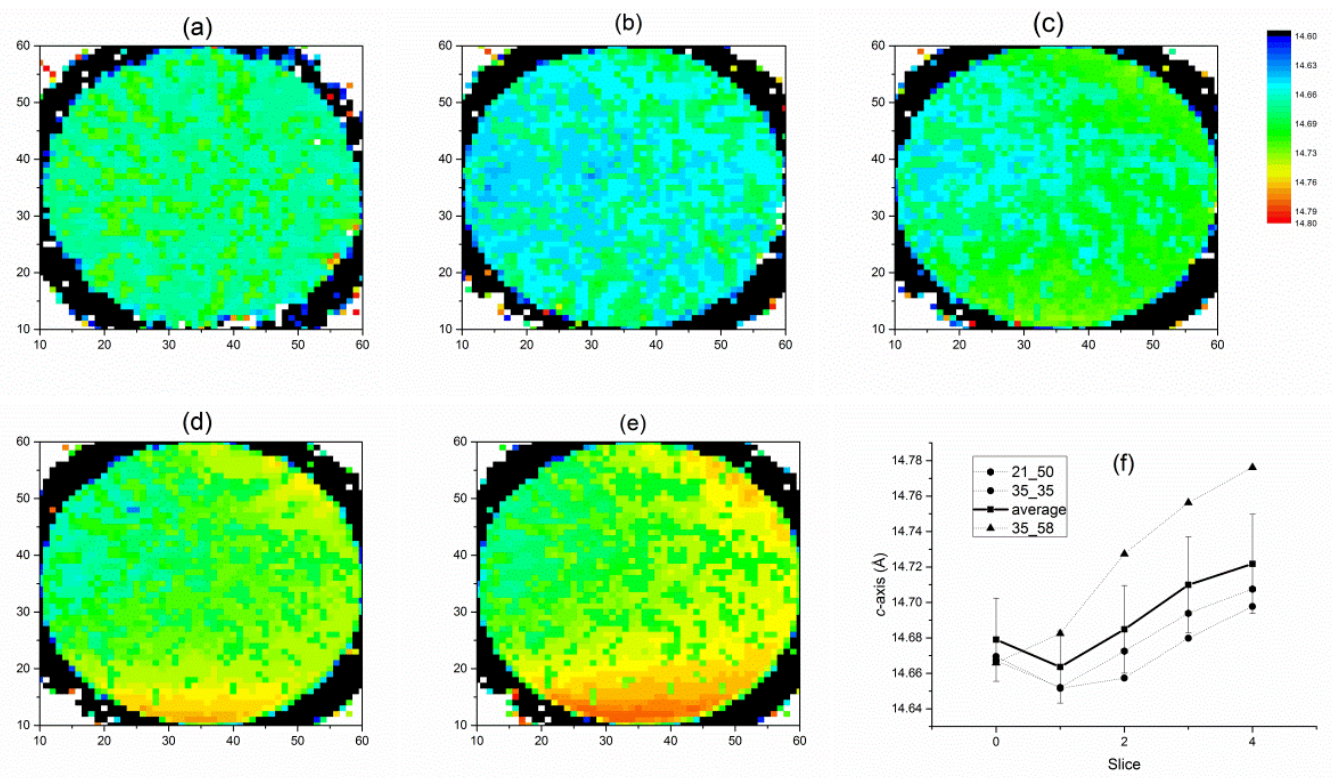
Figure 3 (a-e) Rietveld-CT reconstruction on Rwp of the SAPO-34 (8 \% Si) reactor bed used in the MTO process for 5 minutes. The patterns observed in the $c$-axis reconstruction are not present. (f) Rietveld plot for voxel 35, 35 in layer zero of the Rietveld-CT reconstruction showing the observed, calculated and difference profiles.
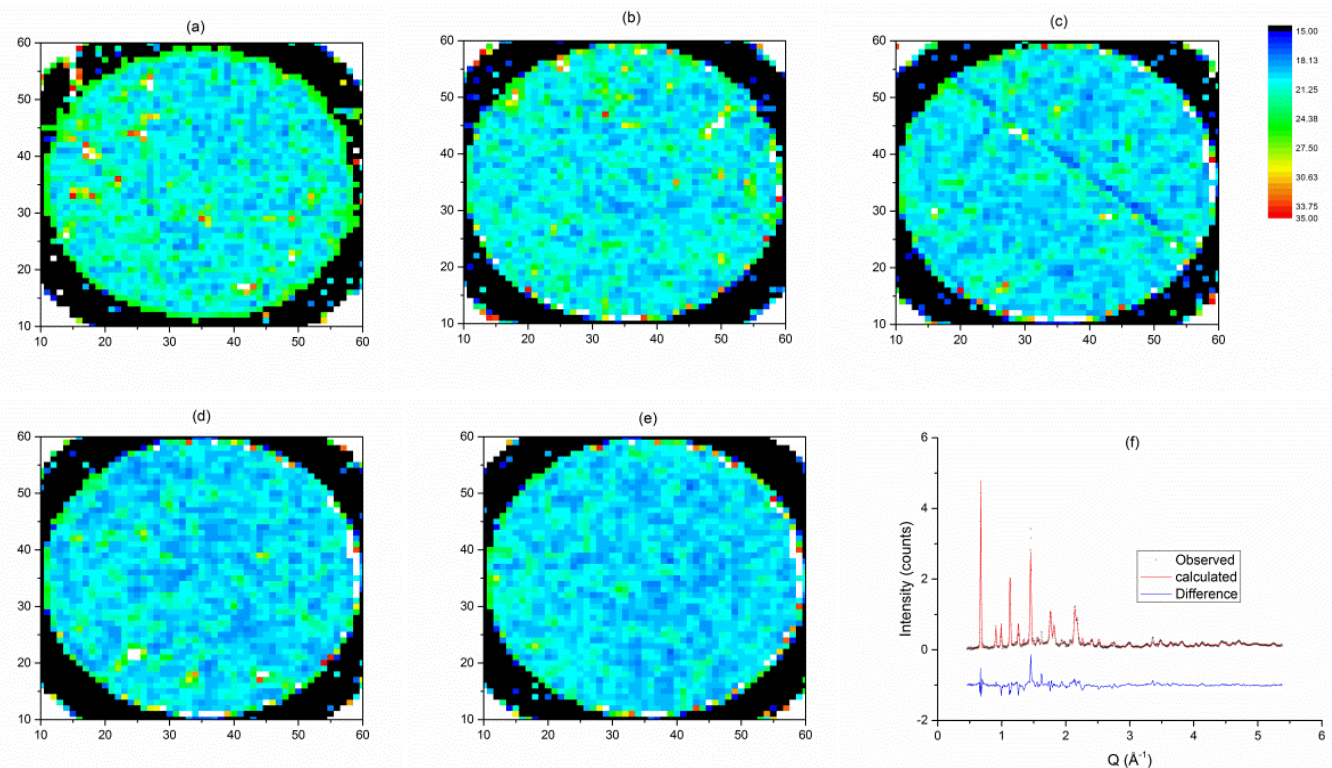
Figure 4 (a-e) Colour contour plots of the $c$-axis Rietveld-CT reconstruction of a SAPO-34 (8 \% Si) reactor bed used in the MTO process for 195 minutes. The tomograph consists of five slices 2-D from slice (a) (inlet) to slice (e) (outlet). The spotty area on the left hand side of slice (e) is due to the fact that the sample moved during data collection leaving this part of the reactor empty, values in slice (d) are also affected. The slices appear somewhat smaller than those for the other samples as a larger dataset was used in the refinement due to the fact that the sample moved. The colour scale used to indicate the $c$-axis expansion is shown in the top right corner. (f) is a plot of c-axis values for selected voxels in each slice (dotted lines) with the average value (heavy line) for comparison. Slice numbers 0-4 in (f) correspond to slices a-e.
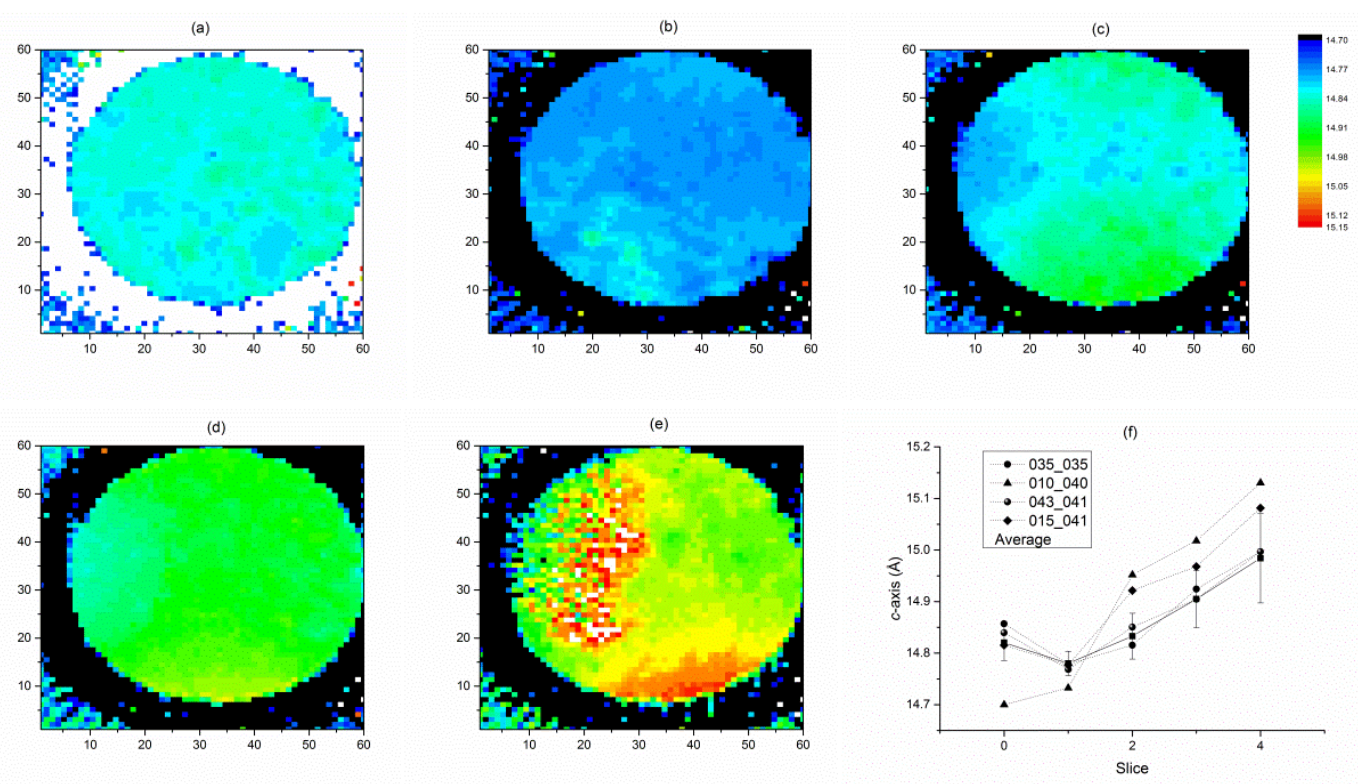
Figure 5 Comparison of “normal” XRD-CT reconstructions (at $Q=0.8088 \AA^{-1}$, the position of the strongest peak in the powder patterns) for slice (e) of the fully reacted (left) and quenched (right) 8\% Si SAPO-34 samples. The very low intensity of the fully reacted slice makes it very hard to compare with the quenched one. Rietveld-CT reconstruction (see above) shows that the two have closely related patterns of $c$-axis expansion. Note that the fully reacted slice XRD-CT shown is upside down compared to the Rietveld reconstruction of the same sample.

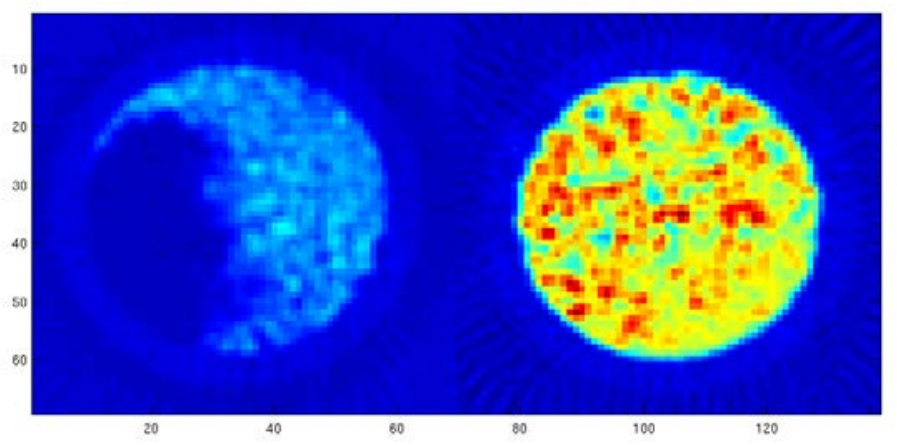

Figure 6 (a-e) Colour contour plots of the $a$-axis Rietveld-CT reconstruction of a SAPO-34 (8 \% Si) reactor bed used in the MTO process for 195 minutes. The tomograph consists of five slices 2-D from slice (a) (inlet) to slice (e) (outlet). The spotty area on the left hand side of slice (e) is due to the fact that the sample moved during data collection leaving this part of the reactor empty, values in slice (d) are also affected. The colour scale used to indicate the $a$-axis length is shown in the top right corner. (f) Is a plot of averaged $a$-axis values each slice. Slice numbers 0-4 in (f) correspond to slices a-e.
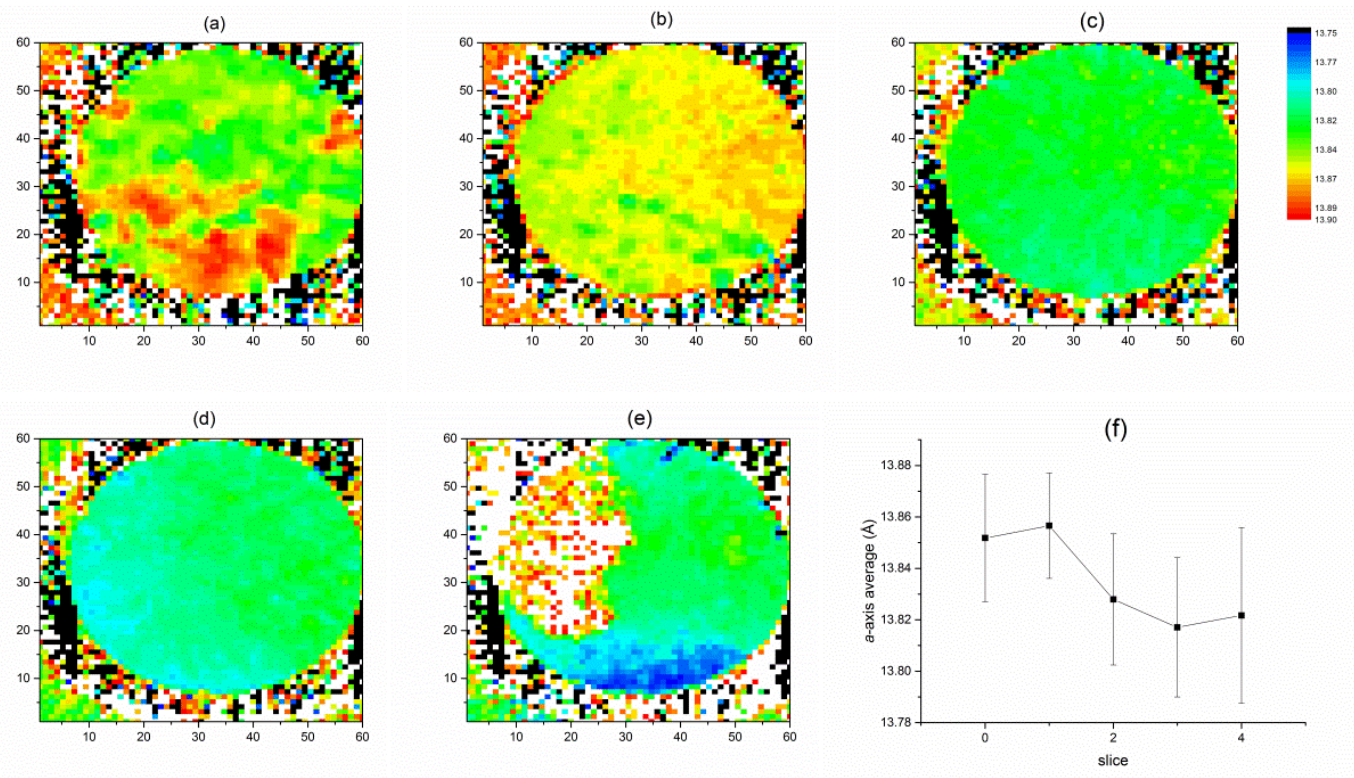
Figure 7 Reconstructions of slice 0 for fully reacted $8 \%$ silicon SAPO-34, note the similarity between the occupancy and $a$-axis plots. The colour range used in the $c$-axis plot has been altered relative to the other plots (14.7-14.9 $\AA$ ) to give better contrast.
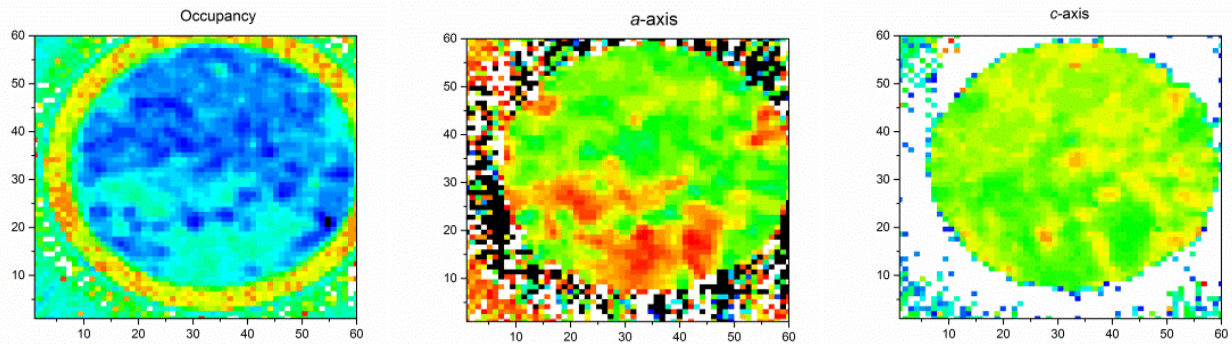

Figure 8 (a-e) Colour contour plots of the $c$-axis Rietveld-CT reconstruction of a SAPO-34 (4 \% Si) reactor bed used in the MTO process for 230 minutes. The tomograph consists of five slices 2-D from slice (a) (inlet) to slice (e) (outlet). The colour scale used to indicate the $c$-axis expansion is shown in the top right corner. (f) is a plot of c-axis values for selected voxels in each slice (dotted lines) with the average value (heavy line) for comparison. Slice numbers 0-4 in (f) correspond to slices a-e.
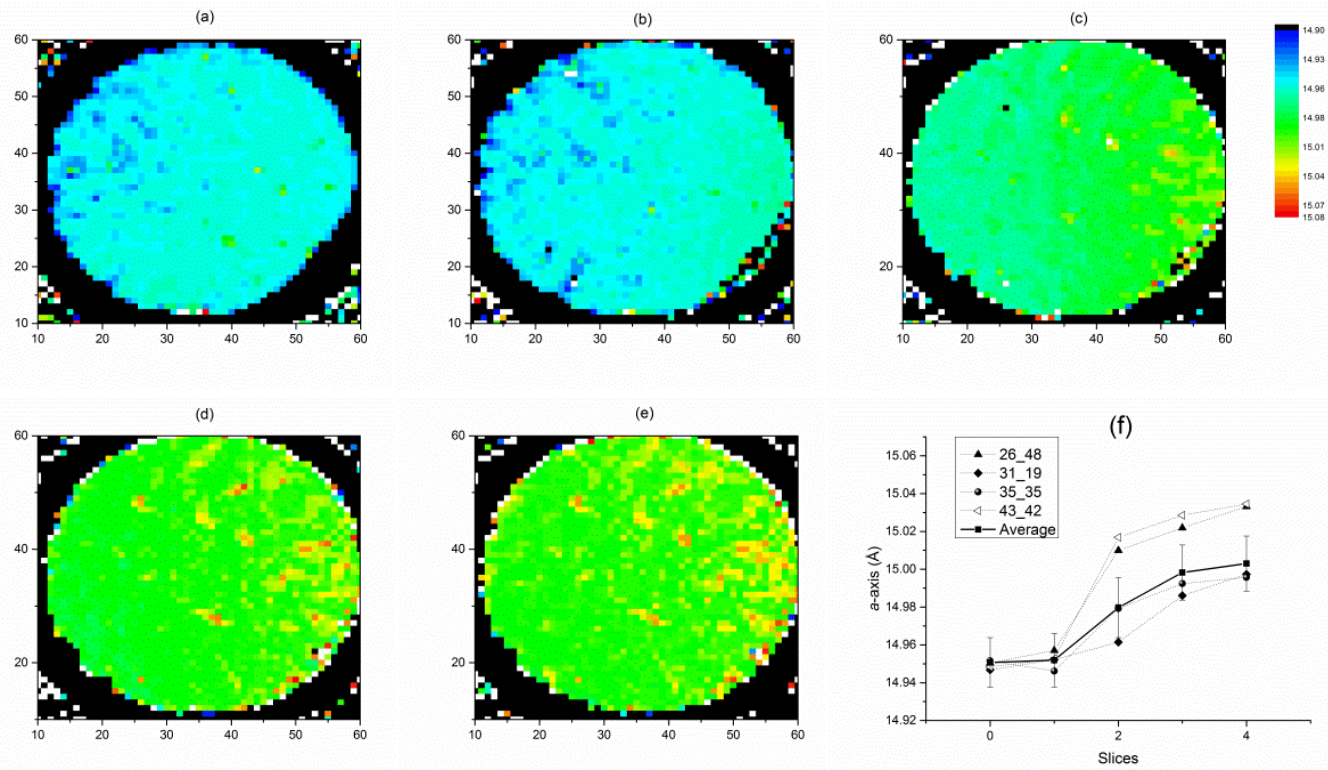
Figure 9 Average $c$-axis variations for the slices of the Rietveld-CT reconstructions. Slice numbers 0-4 in (f) correspond to slices a-e as for figures 3 and 5.

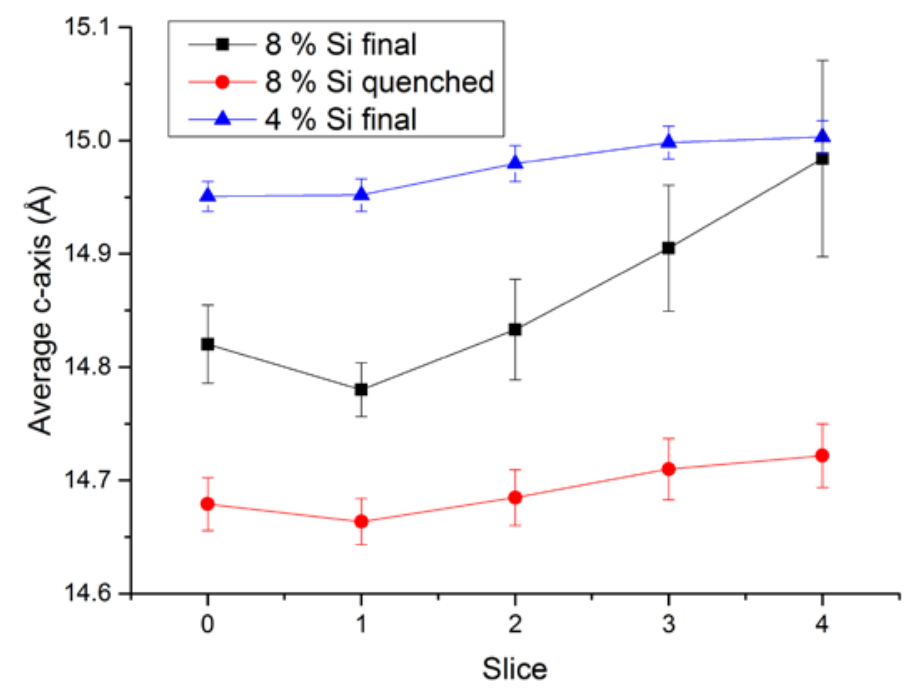

Figure 10 Average cage occupancy values for the five slices of the tomographs for $8 \% \mathrm{Si}$ SAPO-34 (fully reacted and quenched after 5 minutes of reaction) and $4 \%$ Si SAPO-34 (fully reacted). Slice numbers 0-4 correspond to slices a-e.

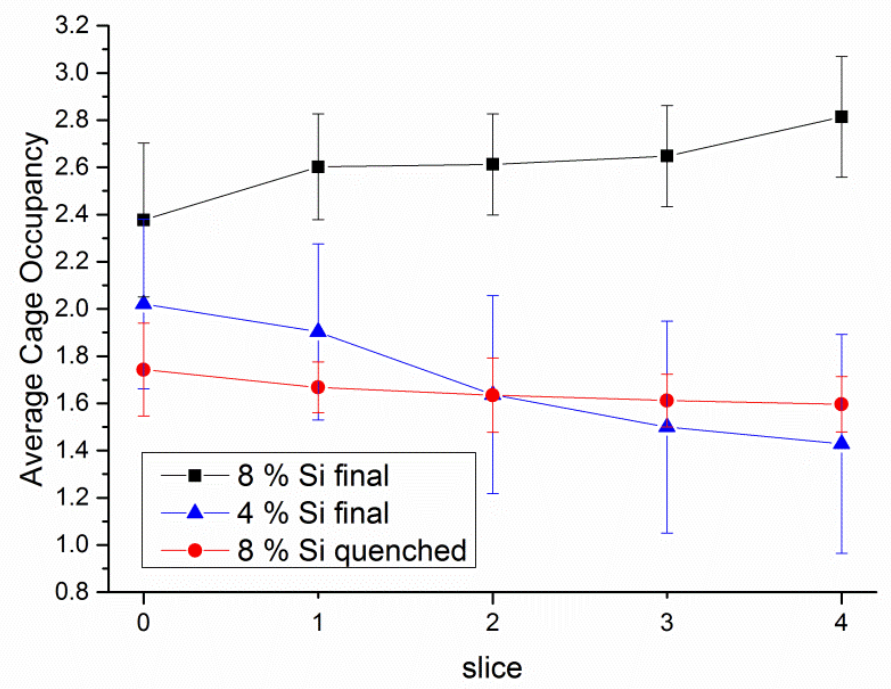




\section{Supporting information}

S1. Refinement details/ fitting procedure The Rietveld refinements presented were carried out using TOPAS academic version 4.2. Initial structural parameters from the SAPO-34 framework and carbon atoms placed on at positions where electron density peaks were observed in the cages in high resolution XRD studies were refined against the powder pattern of the central voxel of slice 0 for the relevant tomograph to obtain good starting values. A parametric Rietveld input file was then prepared using a simple text replacement script to copy the starting parameters into an input file which could be used to treat the powder patterns from the whole slice simultaneously. The command conserve_memory was added to the top of the input file before running to supress graphical output. Thus command should always be used when dealing with very large datasets such as these. An extract from a typical parametric input file is shown below. “...” indicates truncation of the input file. Complete input for one powder pattern is shown along with the essential initial "conserve_memory" command at the top of the file and continuation into the input for the next powder pattern at the end:

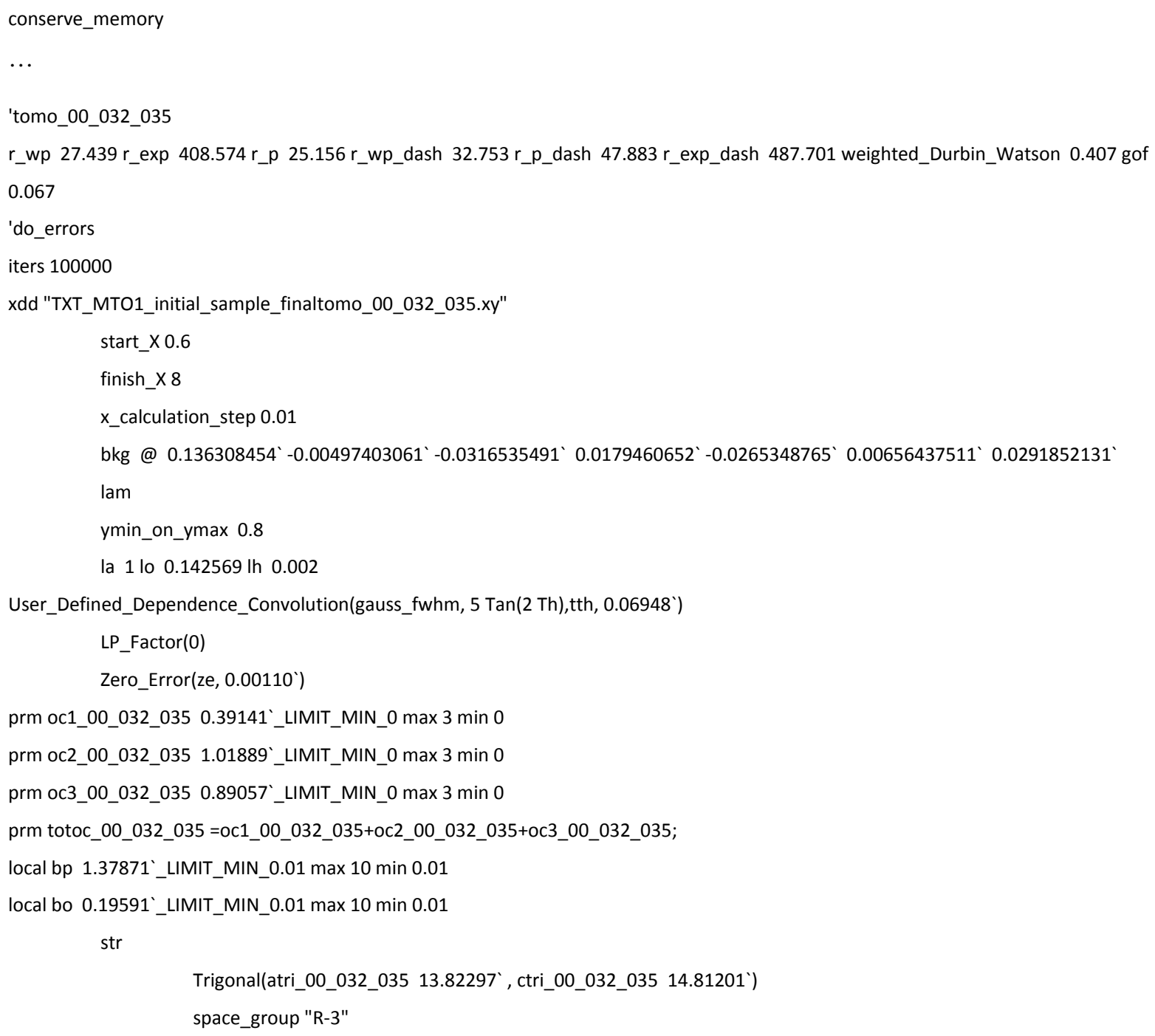




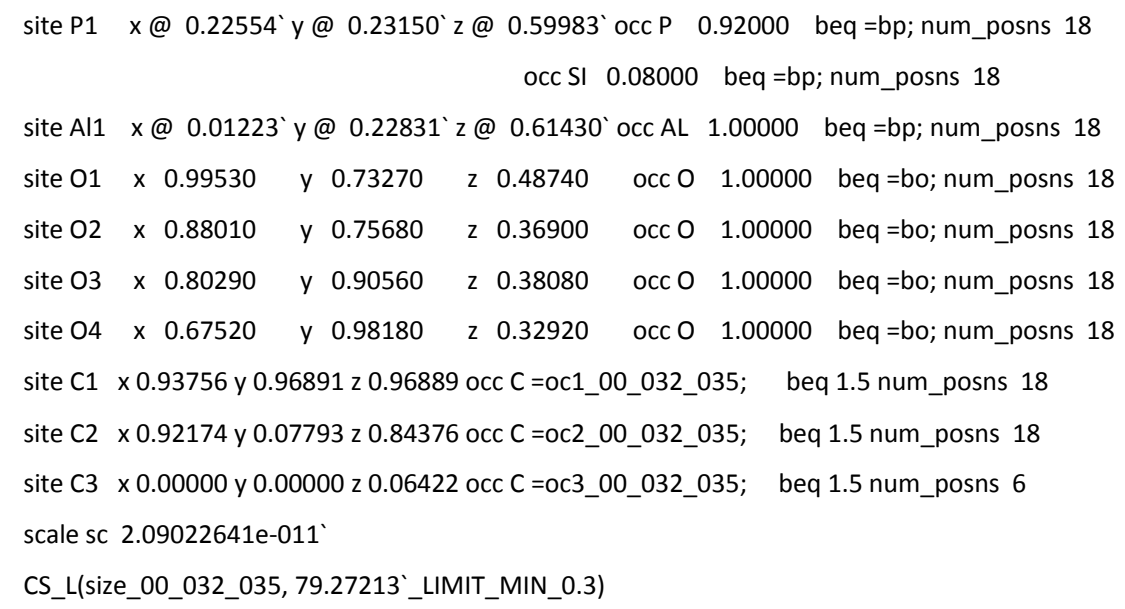

Out_Yobs_Ycalc_and_Difference("MTO1_initial_sample_finaltomo_00_032_035.xyd")

Out_X_Yobs("MTO1_initial_sample_finaltomo_00_032_035.xy")

out "delme.txt" append

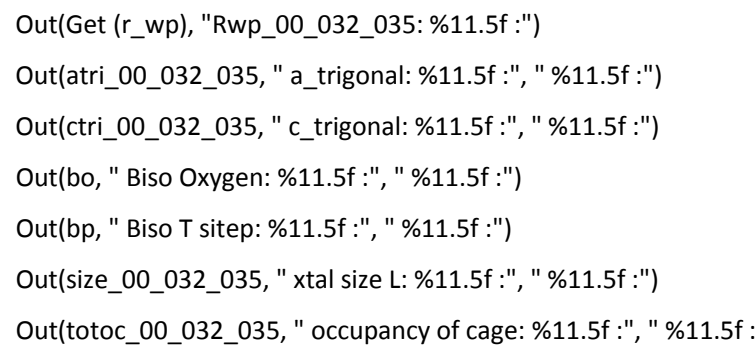

")

'al bond length restraints

Distance_Restrain(Al1 O3 $\left.2 \quad 100-1,1.73,1.62277^{\circ}, 0,500\right)$

Distance_Restrain(Al1 $025-1-1-1,1.73,1.41855^{\circ}, 0,500$ )

Distance_Restrain(Al1 $\left.015-1-1-1,1.73,1.62063^{\circ}, 0,500\right)$

Distance_Restrain(Al1 $\left.042 \quad 10-1,1.73,1.58233^{\prime}, 0,500\right)$

'P bond length restraints

Distance_Restrain(P1 O1 $\left.3 \quad-1 \quad 0 \quad 0,1.53,1.74449^{\prime}, 0,500\right)$

Distance_Restrain(P1 035 -1 -1 -1, 1.53, 1.75596', 0, 500)

Distance_Restrain(P1 O4 1201 0, 1.53, 1.71044', 0, 500)

Distance_Restrain(P1 $\left.025-1-1-1,1.53,1.61488^{\prime}, 0,500\right)$

'OPO angle restraints

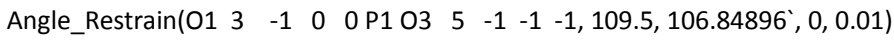

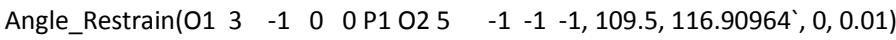

Angle_Restrain(013 3 -1 000 P1 O4 $\left.120010,109.5,105.56820^{\circ}, 0,0.01\right)$

Angle_Restrain(02 5 -1 -1 -1 P1 035 -1 -1 -1, 109.5, 109.61330', 0, 0.01)

Angle_Restrain(02 5 -1 -1 -1 P1 0412012 0, 109.5, 111.63363', 0, 0.01)

Angle_Restrain(04 $12 \quad 0 \quad 10$ P1 O3 5 -1 -1 -1, 109.5, 105.56103', 0, 0.01)

'oalo angle restraints

Angle_Restrain(02 5 -1 -1 -1 Al1 032210 -1, 109.5, 118.24333', 0, 0.01)

Angle_Restrain(02 5 -1 $-1-1$ Al1 $\left.015 \quad-1-1-1,109.5,110.55183^{\prime}, 0,0.01\right)$

Angle_Restrain(02 5 -1 -1 -1 Al1 O4 $2 \quad 10$-1, 109.5, 115.27275', 0, 0.01)

Angle_Restrain(03 $2 \quad 1 \quad 0-1$ Al1 $015 \quad-1-1-1,109.5,102.85061^{\prime}, 0,0.01$ )

Angle_Restrain(03 $2 \quad 100-1$ Al1 $042 \quad 1 \quad 0-1,109.5,107.35260^{\circ}, 0,0.01$ )

Angle_Restrain(01 $5 \quad-1 \quad-1-1$ Al1 $\left.042 \quad 100-1,109.5,100.42368^{\prime}, 0,0.01\right)$ 
xo_Is

xo x_glass 2.33334059' I @ 6.01803694e-006'_LIMIT_MIN_1e-010

CS_L(, 9.90666)

'tomo_00_032_036

r_wp 18.855 r_exp 272.213 r_p 15.289 r_wp_dash 24.356 r_p_dash 30.379 r_exp_dash 351.622 weighted_Durbin_Watson 0.580 gof 0.069

'do_errors

iters 100000

xdd "TXT_MTO1_initial_sample_finaltomo_00_032_036.xy"

$\cdots$

Table S1 lists the refined parameters and how they were treated (independent to each powder pattern or simultaneous refinement against all patterns in the parametric input file). 
Table S1 Rietveld refinement parameters and refinement methods

\begin{tabular}{|c|c|c|c|c|}
\hline \multirow{3}{*}{ Parameter } & \multirow{3}{*}{$\begin{array}{l}\text { Number of } \\
\text { parameters/powder } \\
\text { pattern }\end{array}$} & \multicolumn{3}{|c|}{ How refined? } \\
\hline & & $8 \% \mathrm{Si}$ & 8 \% Si Fully & $4 \%$ Si Fully \\
\hline & & Quench & Reacted & Reacted \\
\hline \multirow{2}{*}{ Background } & \multirow{2}{*}{7} & Individual & Individual & Individual \\
\hline & & Pattern & Pattern & Pattern \\
\hline Tan theta Broadening & 1 & All Data & All Data & All Data \\
\hline Zero error & 1 & All Data & All Data & All Data \\
\hline \multirow{2}{*}{ Dummy carbon occupancy } & \multirow{2}{*}{3} & Individual & Individual & Individual \\
\hline & & Pattern & Pattern & Pattern \\
\hline \multirow{2}{*}{ Biso Phosphorus/aluminium } & \multirow{2}{*}{1} & Individual & Individual & \multirow{2}{*}{ Fixed } \\
\hline & & Pattern* & Pattern* & \\
\hline \multirow{2}{*}{ Biso Oxygen } & \multirow{2}{*}{1} & Individual & Individual & \multirow{2}{*}{ Fixed } \\
\hline & & Pattern* & Pattern* & \\
\hline \multirow{2}{*}{$\mathrm{x}, \mathrm{y}, \mathrm{z}$ phosphorus } & \multirow{2}{*}{3} & Individual & Individual & Individual \\
\hline & & Pattern & Pattern & Pattern \\
\hline \multirow{2}{*}{$\mathrm{x}, \mathrm{y}, \mathrm{z}$ aluminium } & \multirow{2}{*}{3} & Individual & Individual & Individual \\
\hline & & Pattern & Pattern & Pattern \\
\hline Scale & 1 & All Data & All Data & All Data \\
\hline \multirow{2}{*}{ Lorentzian peak broadening } & \multirow{2}{*}{1} & Individual & Individual & Individual \\
\hline & & Pattern & Pattern & Pattern \\
\hline Glass peak position & 1 & All Data & All Data & All Data \\
\hline \multirow{2}{*}{ Glass peak intensity } & \multirow{2}{*}{1} & Individual & Individual & Individual \\
\hline & & Pattern & Pattern & Pattern \\
\hline \multirow{2}{*}{ Glass peak broadening } & \multirow{2}{*}{1} & \multirow{2}{*}{ All Data } & Individual & Individual \\
\hline & & & Pattern & Pattern \\
\hline
\end{tabular}

\footnotetext{
* Refinement of the Biso parameters against all patterns in the tomographic slice was also tested. Maximum and minimum limits of 10 and 1 applied to Biso.
} 
Figure S1 Location of the 5 tomographic slices on a sample reactor bed.

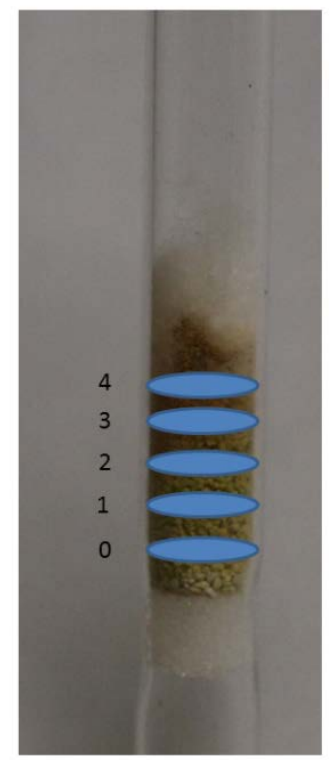

Figure S2 Average Rwp per tomographic slice for the 8\% silicon SAPO-34 quenched after 5 minutes of MTO reaction. Error bars correspond to the standard deviation in the mean average.

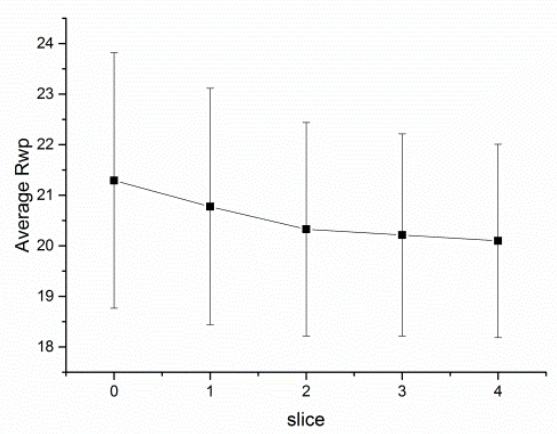


Figure S3 Tomographic slices 0 and 4 for quenched $8 \%$ Si SAPO-34 reconstructed on Biso for the aluminium (left) and phosphorus/silicon (right) sites.

Slice 0
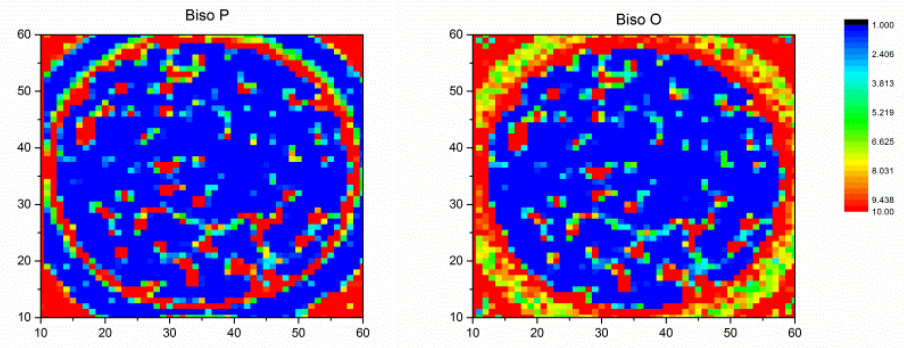

Slice 4
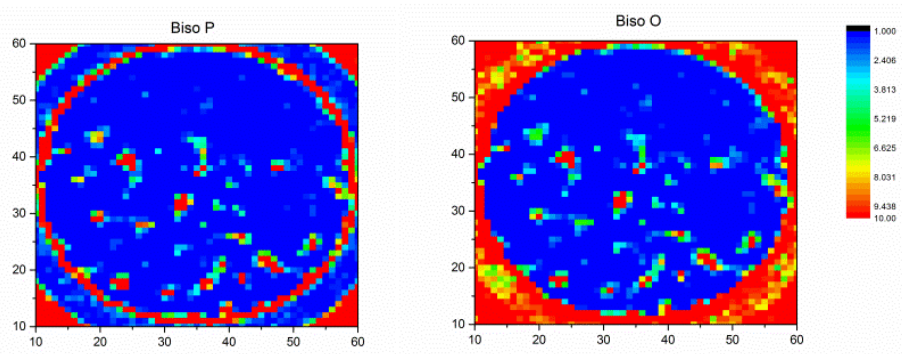
Figure S4 Reconstructions of the quenched $8 \%$ Si SAPO-34 bed on x, y and z coordinates for aluminium (slices 0 and 4 ) and phosphorus (slice 4).
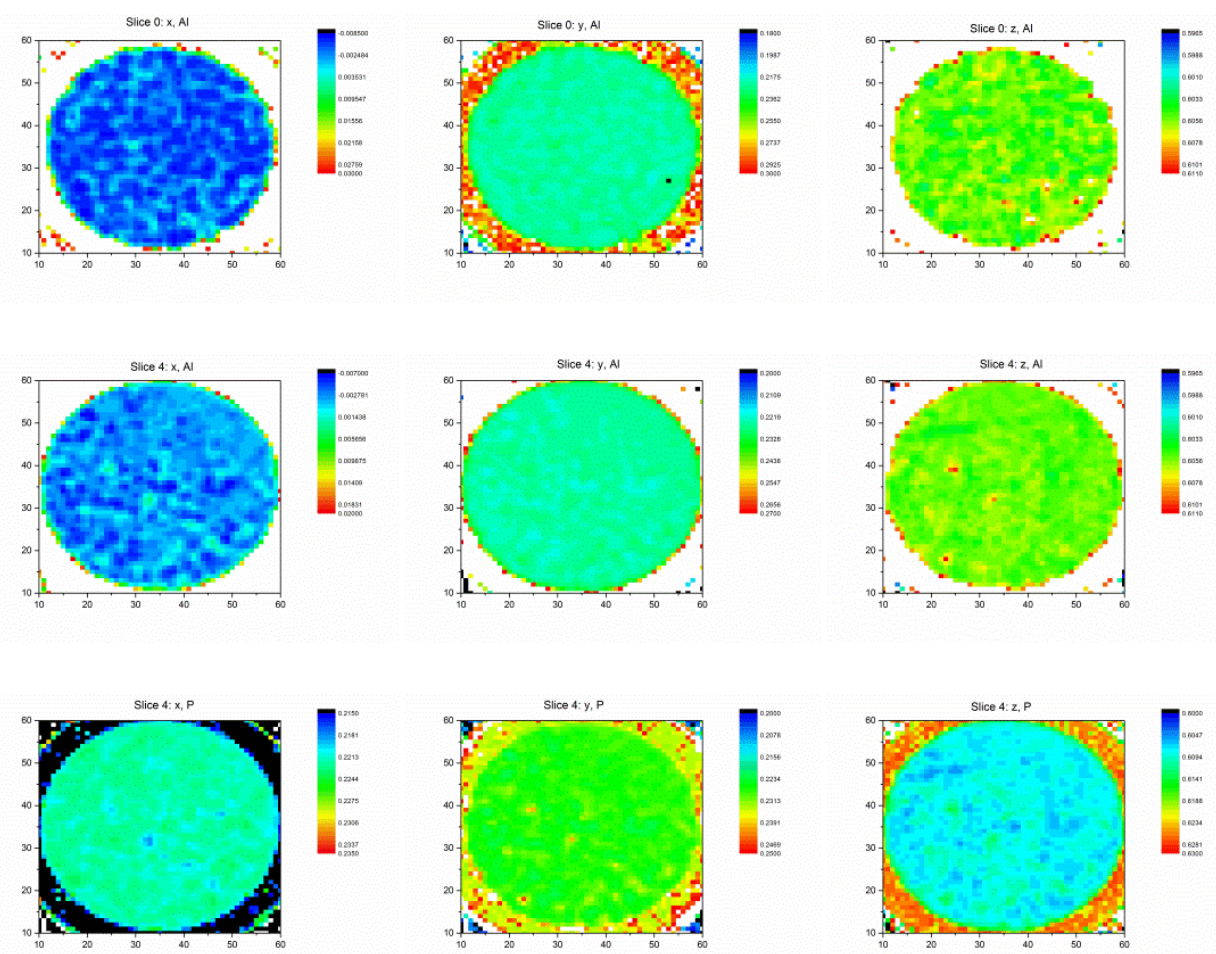

Figure S5 Reconstructions of the quenched $8 \%$ Si SAPO-34 bed on peak broadening.
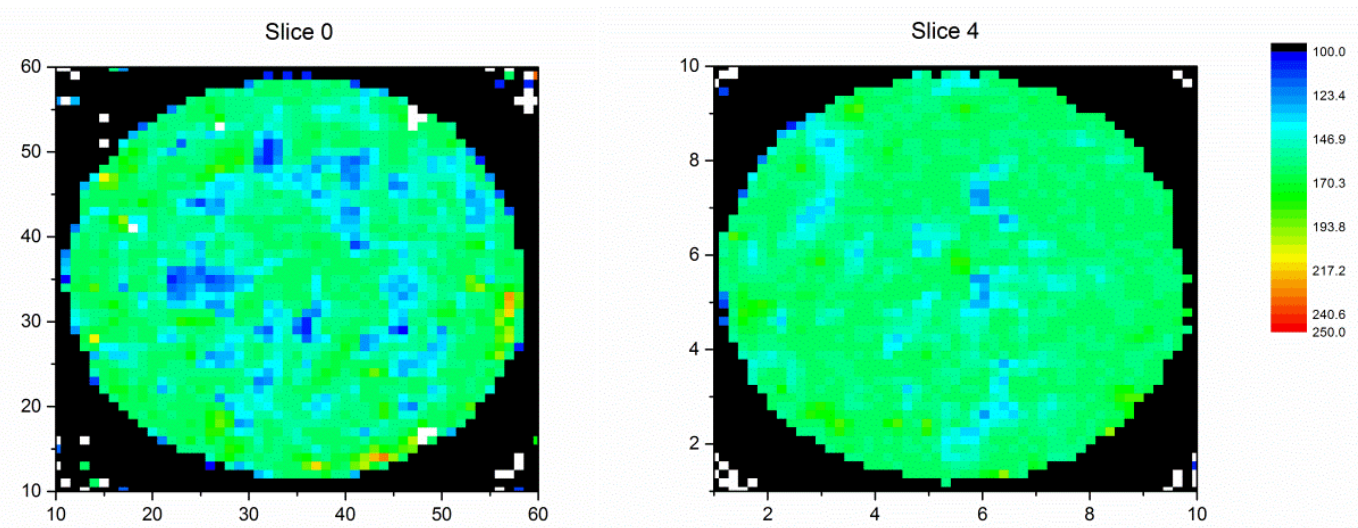
Figure S6 Tomographic slices for fully reacted 8 \% Si SAPO-34 reconstructed on cage occupancy. (a) Slice closest to reactor inlet - (e) slice furthest from inlet.
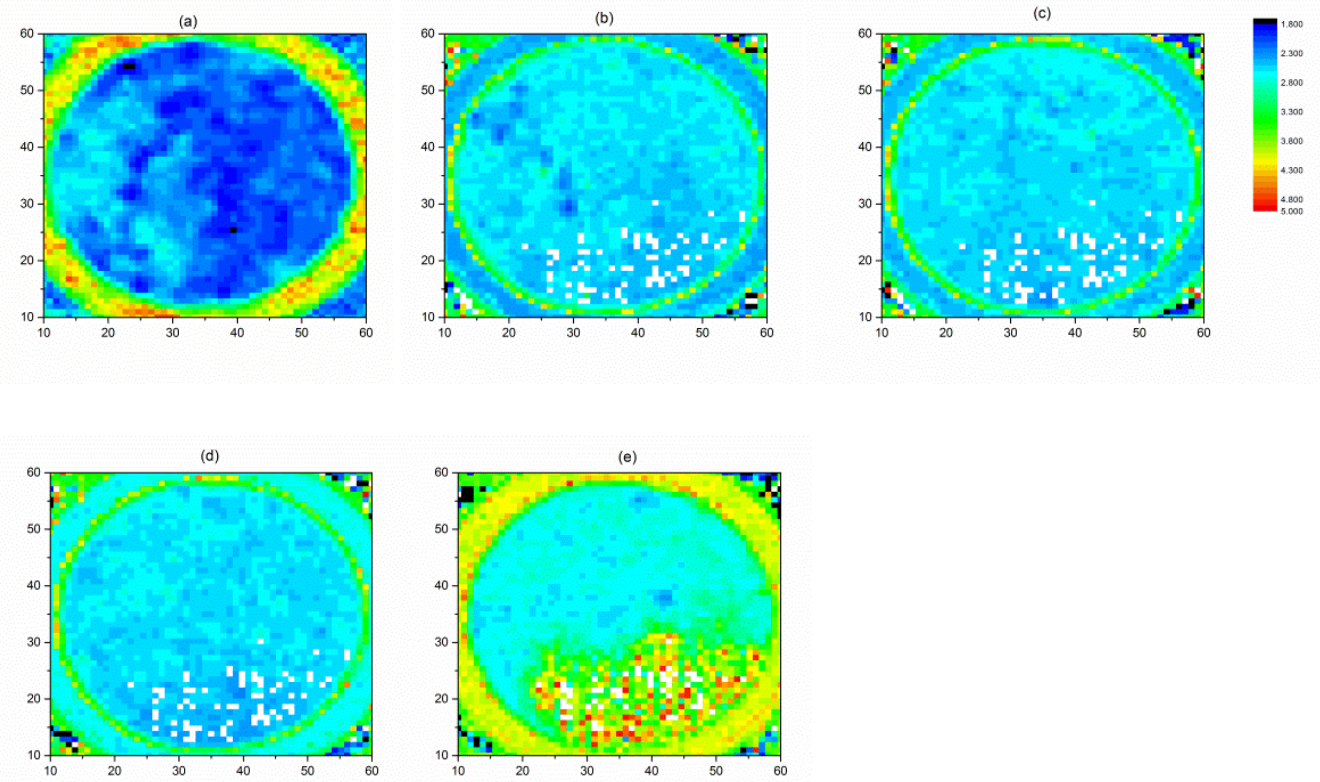

Figure S7 Comparison of the average $c$-axis variations in the tomographic slices compared to the zscan data published previously (Wragg et al., 2012)
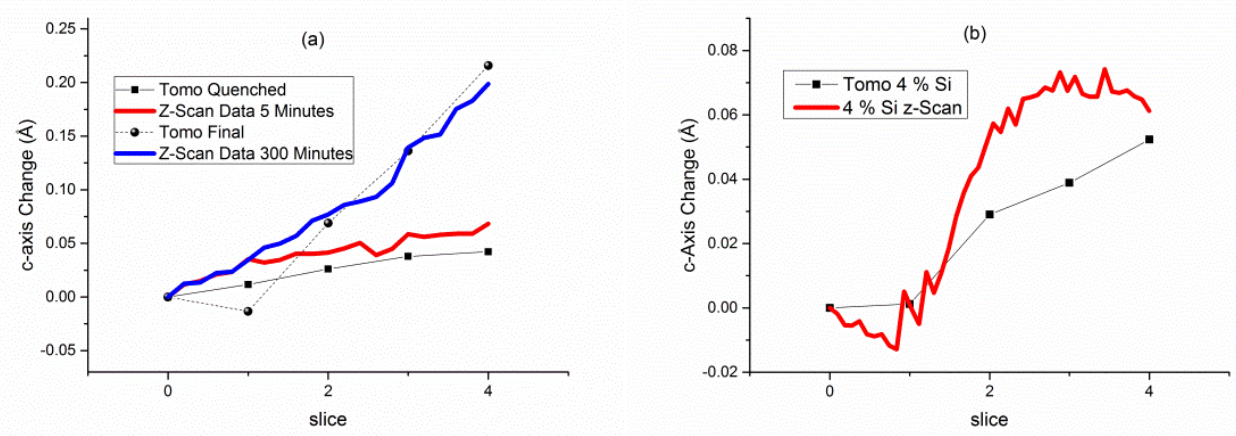